\title{
Radiological and functional characteristics of airway mucociliary clearance
}

\author{
Adolf A Krishtafovich ${ }^{1}$ Zagdyn Zinaida ${ }^{1}$ and Boris M Ariel ${ }^{1,2 *}$ \\ ${ }^{1}$ Federal Institution "Saint-Petersburg Research Institute of Phthisiopulmonology", Healthcare Ministry of Russia: 191036, Ligovskiy pr. 2/4, Saint-Petersburg, Russia \\ ${ }^{2}$ Saint-Petersburg City Bureau of Pathology: 194354, Uchebnyy per. 5, Saint-Petersburg, Russia
}

\begin{abstract}
Aim: The aim of the study was to develop a radiological model for visual assessment of morphology and function of the upper and lower airways ciliated epithelium and the whole mucociliary clearance in healthy subjects and in patients with pulmonary tuberculosis, chronic obstructive pulmonary disease, and lung cancer.

Methods: We examined 65 patients at the age of 36-87 of whom 26 subjects were with limited lung lesions and normal airway mucosa, as the control group, and 39 - with extended lung lesions due to tuberculosis, chronic obstructive pulmonary disease, or lung cancer, as the study group. The airway mucosa functional activity was investigated by spray coating of tantalum microparticles.

Results: In the control group, the time of complete clearance was from 15 to 20 min in larynx and pharynx, 45 to 60 min in trachea and from 1-3 to $20-24$ h in bronchi. Bronchial particles clearance was decreased in the study group. The airway clearance disturbance was considered as moderate in patients with acute inflammation such as in tuberculosis, nonspecific pneumonia, acute bronchitis, etc., severe - in patients with chronic bronchial and pulmonary diseases such as tuberculosis pulmonum fibrosa cavernosa, chronic bronchitis, bronchiectasis, and very severe - in patients with lung cancer. The clearance time in patients with these diseases was ranged from 24 to $48 \mathrm{~h}$, from 48 to $72 \mathrm{~h}$ and $\geq 72 \mathrm{~h}$, respectively.

Conclusion: Normally, the airway clearance rate is in 60-65 times lower in the small airways than in the upper airways (pharynx and larynx). Local clearance disturbance caused by tobacco smoking, pollutants, etc., greatly decreases bronchial mucus transport and prolongs the complete airway clearance time that could provide occurrence and progression of inflammatory and malignant pulmonary diseases.
\end{abstract}

\section{Introduction}

Due to the steady increase in the lung inflammatory and malignant diseases occurrence and their delayed diagnosis the necessity in the deepest learning of morpho functional disturbance of the mucosal ciliated apparatus and airway mucociliary clearance (MC) emerges. In the literature this problem is discussed insufficiently $[1,2]$ because it is impossible to study the mucosal functional activity in a short endoscopy examination in vivo using various markers. The most informative methods are radioisotope [3,4] and hemoglobin [5]. The radioisotope method gives a quantitative assessment of the total particles clearance allowing to establish a certain regularity in the rate of particles transport from the small bronchi to the vocal cords. Thus, it was shown that in the normal mucosa this speed, like an avalanche, increases by $20-25$ times, and the time for complete cleansing of the mucosa varies within 20-24 hours.

It can be assumed that not only quantitative indicators characterize the morpho functional state of the ciliary apparatus of the mucosa and MK in normal and pathological conditions, but also its qualitative features that are not currently detected by traditional endoscopic methods using well-known markers. For a more complete, comprehensive characterization of the MK the structural features of the large bronchi mucosa should take into account. We have earlier found that in the latter there are "risk zones" formed as a result of damage to the epithelium during prolonged exposure of the mucosa with pollutant particles, tobacco smoke and other factors. We assume then that there is a detrimental impact on the uninterrupting transport of the respiratory tract contents in a cranial direction, thereby providing the conditions for the development of inflammatory and neoplastic diseases. In the "risk zones", extended excretory ducts, open cysts of the exocrine glands and cicatricial changes after healing of broncho nodular fistulas are more common [6]. It must be admitted that it is there that the microbiota is concentrated, which can trigger autoinfection, which acquires the fulminant course under certain circumstances, for example, in severe burn injury of the respiratory tract [7].

Our previous studies have shown $[8,9]$ that the respiratory tract mucosa is a sui generis information field, and it is a prerequisite for this study. As a matter of fact, the tantalum microparticles spray coating permits to obtain on the bronchograms a distinct display of individual details of the mucosa relief, such as interfold gaps, irregularities, scar changes, excretory ducts of exocrine glands and their cysts, etc. [9].

It can be assumed that by spraying tantalum microparticles on the mucosa and tracing their transport in dynamics, we will obtain

*Correspondence to: Ariel Boris M, Federal Institution "Saint-Petersburg Research Institute of Phthisiopulmonology", Healthcare Ministry of Russia: 191036, Ligovskiy pr. 2/4, Saint-Petersburg, Russia, Tel: +7 (812) 513-60-98; E-mail: arielboris@rambler.ru

Key words: trachea, bronchi, mucociliary clearance, tantalum microparticles, risk zones, radiological model

Received: March 04, 2019; Accepted: March 19, 2019; Published: March 22, 2019 
objective information on laryngograms and bronchograms, enough to assess the function of the ciliated apparatus of the mucosa and MK at various levels. In turn, this information is essential for understanding the pathogenesis of some respiratory diseases and for their early diagnosis. [9].

Our study is aimed to develop a radiological model of visualization of the morpho functional activity in the mucosal ciliary apparatus of the upper and lower respiratory tract, regional and total MC in normal condition, as well as in cases of tuberculosis, chronic obstructive pulmonary disease (COPD), and lung cancer (LC) by using tantalum microparticles as a marker and a special device for its spraying.

\section{Materials and methods}

All patients aged from 18 to 60 years old, admitted to the Institute hospital during the period of May - November 2018 for differential diagnosis of respiratory tract pathology and tuberculosis treatment were included. Excluded were patients with severe clinical and allergic status. Of 87 patients admitted to the hospital during the study period, 69 subjects have met the inclusion criteria, of whom 4 patients refused their participation in the study. The rest of 65 adopted patients were divided into two groups.

The first (control) group was consisted of 26 patients with limited segmental and subsegmental lung lesions, such as tuberculoma $(n=8)$, infiltration ( $n=9)$, cavity $(n=4)$, cyst $(n=2)$, and peripheral LC $(n=3)$. In these patients any changes in the mucosa and any deformations of the bronchial walls have not been detected during endoscopy examination.

The second (main) group was consisted of 39 patients with widespread pathological changes in the lungs: polysegmental infiltrative tuberculosis $(n=13)$, fibro-cavernous tuberculosis $(n=9)$, chronic bronchitis $(n=7)$, bronchiectasis $(n=4)$, and central LC $(n=6)$. Bronchofibroscopy was performed in all cases, and lesions were found in 30 of 39 patients: bronchus infiltrative tuberculosis $(n=5)$, diffuse endobronchitis $(n=15)$, drainage purulent endobronchitis $(n=4)$, and bronchus cancer $(n=6)$.

To visualize the particles transport along the mucosa of the respiratory tract, a tantalum powder usually using for bronchography was used as a marker [4] (Resolution of the Pharmacological Committee of Health Ministry of USSR, dated on 06.25.1992, No 11 "Resolution about using a tantalum powder in the clinic as a radiopaque agent for the tracheobronchography"). Developer is Ulbinsk Metallurgical Factory (Ust-Kamenogorsk, Russia). To visualize the particles transport along the mucosa of the respiratory tract, microparticles of tantalum in an amount of 5-8 g were administered dosed through a catheter using a "Device for inhalation of powdered preparations" (Pat. Of the Russian Federation for the invention № 2053801) [10].

According to the task of the study, the regional and general MC were studied. To study the first catheter was introduced in anatomically isolated areas - the larynx, pharynx, trachea, main and lobe bronchi, etc. To study the general MC catheter was introduced into the trachea. $\mathrm{X}$-ray examination was carried out immediately after spraying and in dynamics after 10-20 min, $1 \mathrm{~h}, 3 \mathrm{~h}, 5 \mathrm{~h}, 24 \mathrm{~h}, 48 \mathrm{~h}$, and $72 \mathrm{~h}$. In order to reduce the radiation load, radiographs were performed selectively, which allowed to reduce their number to 2-3 and no more in each patient. There were no complications associated with the spraying procedure.

After admitting to the hospital, the blood and urine tests, bio-chemical analysis, chest X-ray, sputum and bronchial lavage bacteriological tests for Mycobacterium tuberculosis, and bronchial endoscopy were performed in all cases. The study was allowed by the Ethical Committee of the Scientific Research Institute of Phthisiopulmonology. Before participation in the study a written consent has been signed by all of the patients.

\section{Results and discussion}

In the first, control group (26 patients) the introduction of the marker into the larynx and pharynx lumen and the piriform sinuses has shown that in 3 cases up to $95 \%$ of the inhaled particles were deposited on the mucosa and only $5 \%$ of the ss, mostly small, penetrated into the infraglottic cavity of the larynx (Figure 1A). Deposition of particles was carried out by turbulent vortices with their multilayer deposition at collision with the mucosa, which was confirmed by $\mathrm{x}$-ray plots of high optical density. It should be assumed that these areas are most susceptible to mechanical and toxic effects of various particles and other pathogenic factors, being "risk zones", where inflammatory and tumor processes develop more often.

10 minutes after spraying, it is clearly visible that almost $50 \%$ of the mucosal area has been cleared of particles (Figure 1B). At the same time, their retrograde transport in the form of curved linear flows in the direction of piriform sinuses, which are a kind of depot of such particles, was clearly traced. Complete clearance of the mucosa from the tantalum particles was completed within 15-20 minutes by swallowing the particles and getting them into the stomach (Figure 1C).

Thus, objectively proven retrograde particles transport and the time of complete mucosal cleansing can be a benchmark in the study of the regional MC in patients with tumor and inflammatory diseases of the pharynx and larynx. The use of $1 \%$ lidocaine in the amount of 3-5 ml for local anesthesia did not have a significant effect on the transport of particles.

In the study of the trachea (5 patients), the catheter was installed in the infraglottic cavity (Figure 2A). 10 minutes after spraying, the architectonics of the mucosa relief changed, where spiral flows were formed, directed to the larynx (Figure 2B). At the same time, the speed of particles transport along the tracheal mucosa slowed down by almost 3 times compared to the pharynx and larynx. Complete cleansing of the trachea was completed within 45-60 minutes.

In the main and intermediate bronchi (5 patients) the particles were deposited after spraying mainly in the interstitial spaces of the membranous part of the bronchi and in the "risk zones" (Figure 3A). 7-10 min after spraying areas of different size and shape, free from the particles, were clearly visible in these areas (Figure 3B). It can be assumed the point is that there are metachronous fields with a distinct demarcation line $[7,8,10]$. In the process of transport, the particles merged into separate conglomerates of different size and shape, form 2 streams up to $5 \mathrm{~mm}$ wide, going through the "risk zones" into the trachea. Transport of particles was slowed down, so that the whole cleansing process of the respiratory tract mucosa was completed within 1-2 hours.

In the lobar and segmental bronchi (4 patients), the transported microparticles also merged into conglomerates, which formed streams in the places of bronchial divisions and were sent to the main bronchi. In this case, the transport of particles in the bronchi was even slower than in the larynx and trachea, and the time of complete clearance of the mucosa ranged from 3 to 5 hours. Our observations have shown that there were sometimes located cysts of the exocrine glands and the mouths of the enlarged excretory ducts on the paths of particles transport. 
A

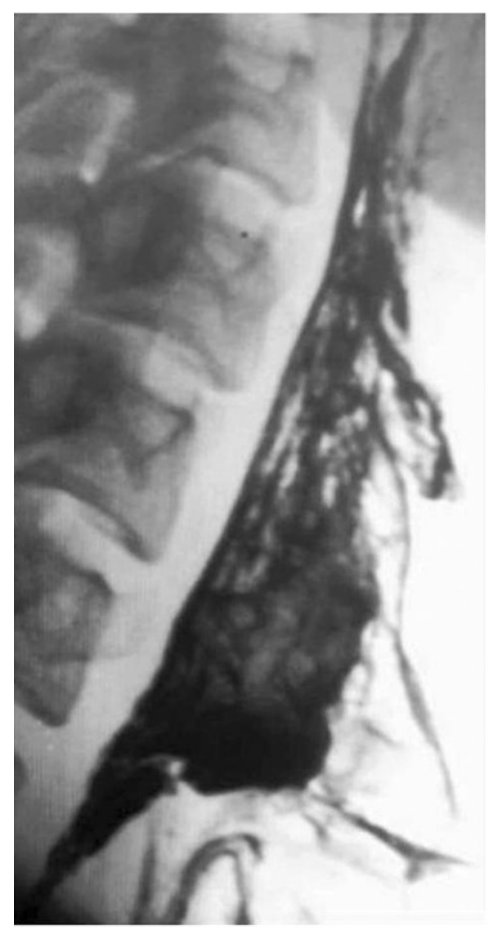

B

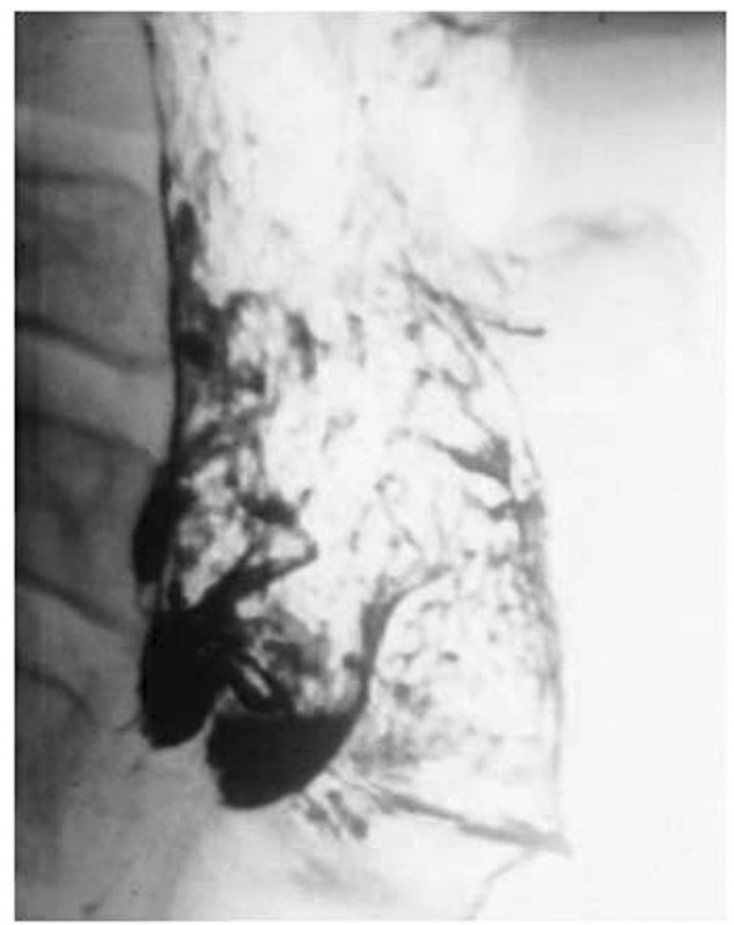

C

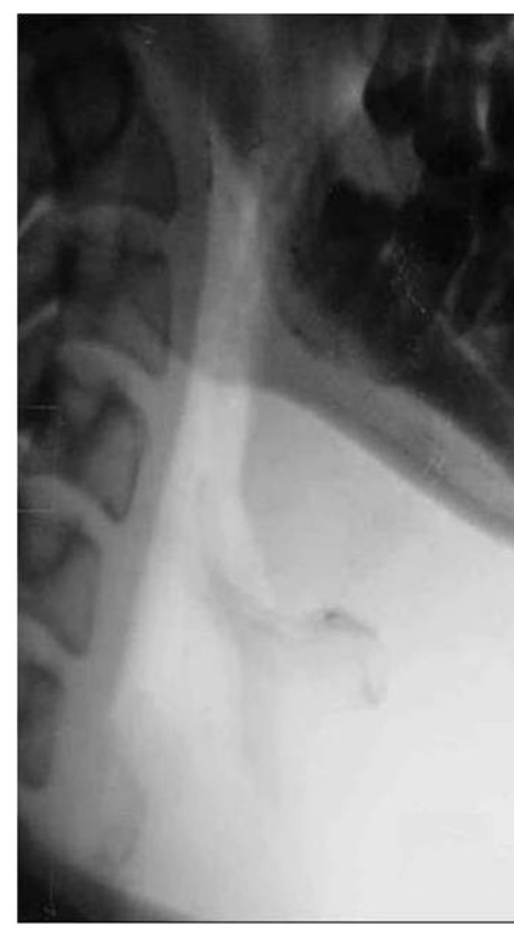

Figure 1. The control group. Pharynx: (A) immediately after the spray coating with tantalum microparticles; B) in 10 min after the spray coating. $50 \%$ clearance of the airway mucosa is seen. There is linear particles flow towards the piriform sinuses; C) in 15 min after the spray coating. The complete clearance of tantalum microparticles is seen.

A

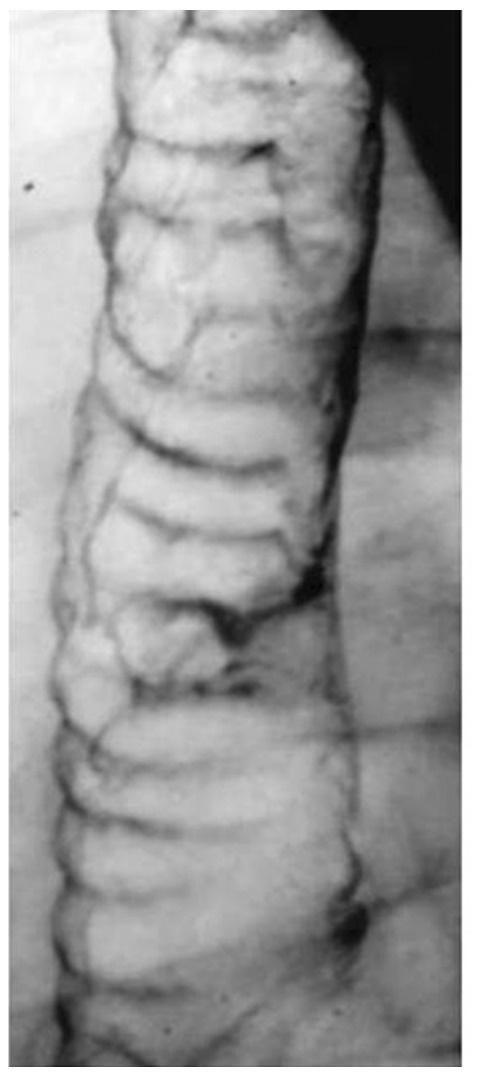

B

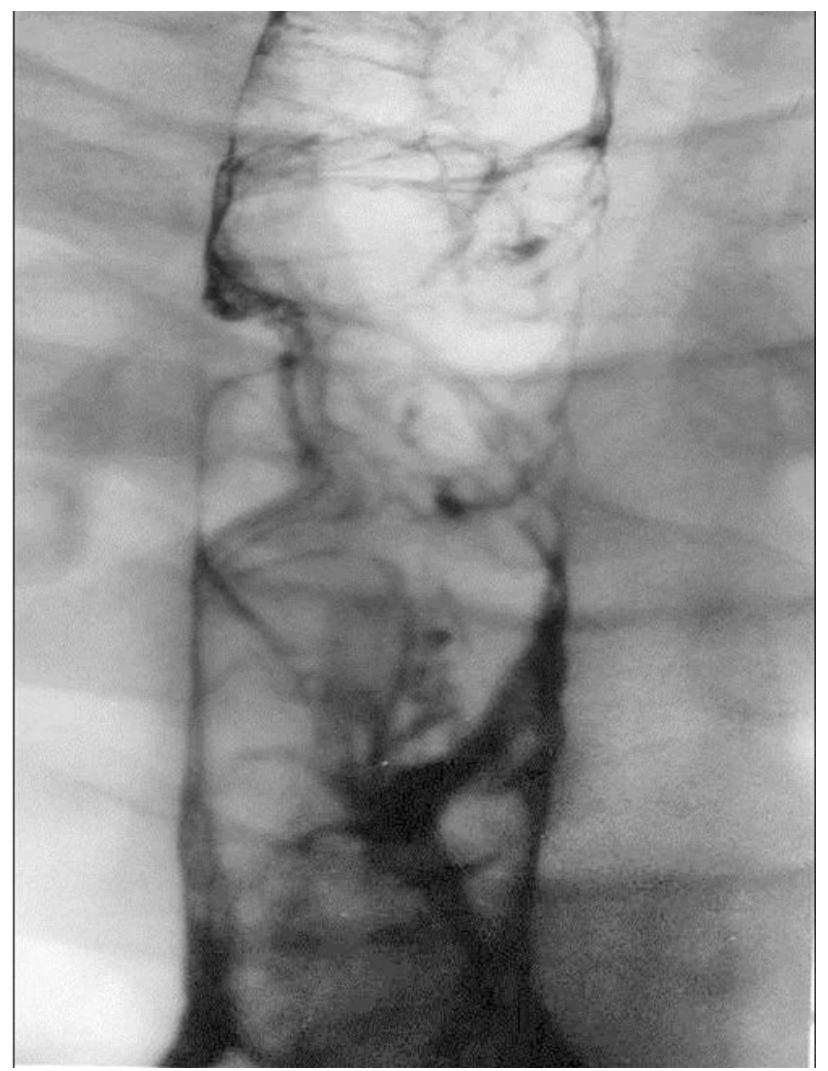

Figure 2. The control group. Trachea. A) a catheter is seen in the infraglottic cavity; B) spiral flow of the particles towards the larynx. 


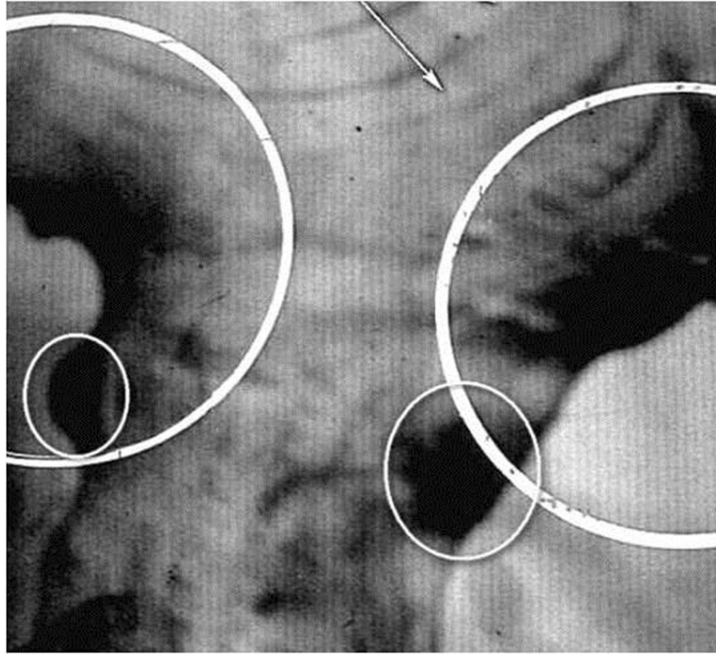

A

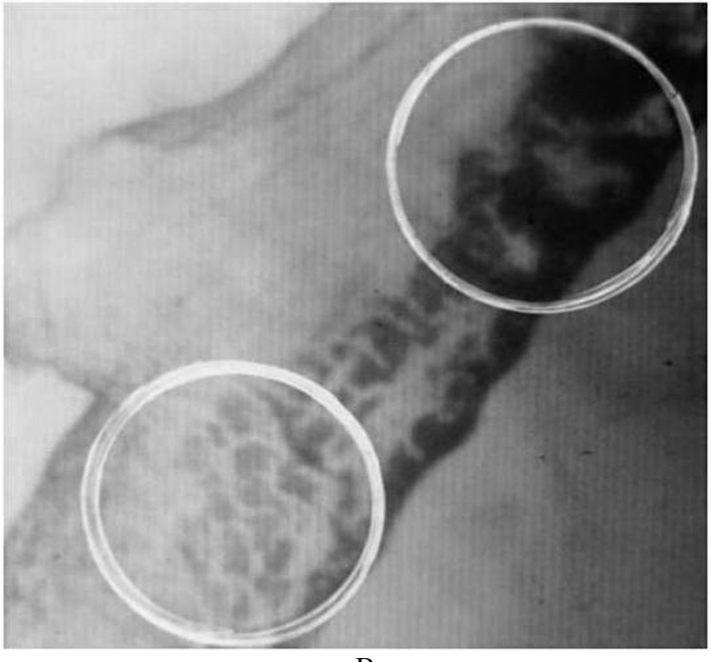

B

Figure 3. The control group. The main bronchus. A) tantalum microparticles are seen in the membranous part of the bronchus and in "risk zones" immediately after the spray coating; B) free of the tantalum microparticles mucosa areas are seen in $10 \mathrm{~min}$ after the spray coating.

Table 1. Tantalum particles transport in the airway mucosa of the $2^{\text {nd }}$ group patients

\begin{tabular}{|c|c|c|c|c|c|c|c|}
\hline \multirow{2}{*}{$\begin{array}{l}\text { Clinical } \\
\text { diagnosis }\end{array}$} & \multirow{2}{*}{$\begin{array}{c}\text { Number of } \\
\text { patients }\end{array}$} & \multicolumn{4}{|c|}{ Clearance time (h) } & \multicolumn{2}{|c|}{ Endoscopy picture } \\
\hline & & $0-24$ & 24-48 & 48-72 & $\geq 72$ & Normal & Pathological \\
\hline Tuberculosis & 22 & 8 & 14 & - & - & 4 & 18 \\
\hline Chronic bronchitis, bronchiectasis & 11 & - & 8 & 2 & 1 & - & 11 \\
\hline Lung cancer & 6 & - & - & 2 & 4 & & \\
\hline Total & 39 & 8 & 22 & 4 & 5 & 4 & 29 \\
\hline
\end{tabular}

In the small bronchi (6 patients), especially lower lobe, transport of particles is slowed so dramatically that the process of mucosa cleansing ranged from 20 to 24 hours.

As can be seen from the above, with a decrease in the caliber of the respiratory tract, the differences in the speed of particles transport increased sharply. It should be assumed that this speed gradient reflects naturally the increasing abatement in the regional functional activity of the ciliated apparatus of the mucosa as the diameter of the bronchi decreases. It is also possible that such factors as smoking, mucus viscosity, temperature, etc. also affect the speed of particles transport, which requires further research.

The $2^{\text {nd }}$ (main) group consisted of 39 patients with tuberculosis, COPD and LC (Table 1).

As can be seen from the Table 1, in 31 (70.4\%) patients the clearance time of the mucosa was 24 hours or more, and in 9 patients it exceeded 48 hours. The longest particles delay ( 72 hours or more) was observed in 4 out of 6 patients with LC.

In 14 of 22 patients (63.6\%) with pulmonary tuberculosis the clearance of particles in the bronchi was reduced (within 24-48 hours). In 5 of them, endoscopic examination revealed infiltrative tuberculosis of the bronchus, in 4 - drainage endobronchitis and in 5-diffuse endobronchitis. According to our observations, in fibrous-cavernous pulmonary tuberculosis the particles transport along the mucosa is significantly affected by the displacement of the trachea (Figure $4 \mathrm{~A}$ ).

20 minutes after spraying, the transport of particles in the trachea took the form of a wide flow $(10 \mathrm{~mm})$ along its entire length (Figure 4B). After 30 minutes, it is clearly visible that the transport of particles is carried out along the mucosa of the cartilaginous part of the trachea
(Figure 4C). Perhaps this is due to its lower mobility compared to the membranous part. Complete purification of the trachea from particles in pulmonary tuberculosis occurred after $60 \mathrm{~min}$.

It can be assumed that in case of deformation of the respiratory tract, these or those additional adaptive mechanisms are activated which accelerate the cleansing process due to the wide flow's initiation, as in the trachea.

In COPD (11 patients), the clearance of tantalum particles along the bronchial mucosa was reduced in all cases. In chronic bronchitis (8 patients) such a delay was from 48 to 72 hours and in bronchiectasis (3 patients)-more than 72 hours. Nonspecific diffuse endobronchitis (8 patients) and purulent drainage endobronchitis (3 patients) were diagnosed in endoscopic examination.

In 6 patients with central LC 4 patients had a particle delay of more than 72 hours and 2 patients - 48-72 hours. 72 hours after spraying a delay of tantalum particles over $3 \mathrm{~cm}$ proximal to a cancer tumor with a seemingly unchanged mucosa was noted. We must assume that this delay was due to the peribronchial growth of the tumor, which was confirmed in the histological examination of the removed lung. Therefore, in the absence of visible changes in the bronchus there is every reason to really judge the extent of the tumor spread in the mucosa by the rate of its purification.

Along with a sharp decrease in the tantalum particles clearance at the tumor location, there was also a disturbance of the clearance distal to the tumor with retrograde particles movement into the alveoli. Likely, this is the mechanism of paracancerous inflammatory changes $[8,11]$.

It can be assumed that the apparatus regulating the mobility of the ciliated epithelium is embedded in the submucosal layer. Histological examination revealed nerve trunks there that penetrate the epithelium and form a kind of terminals between the epithelial cells [12]. 
A

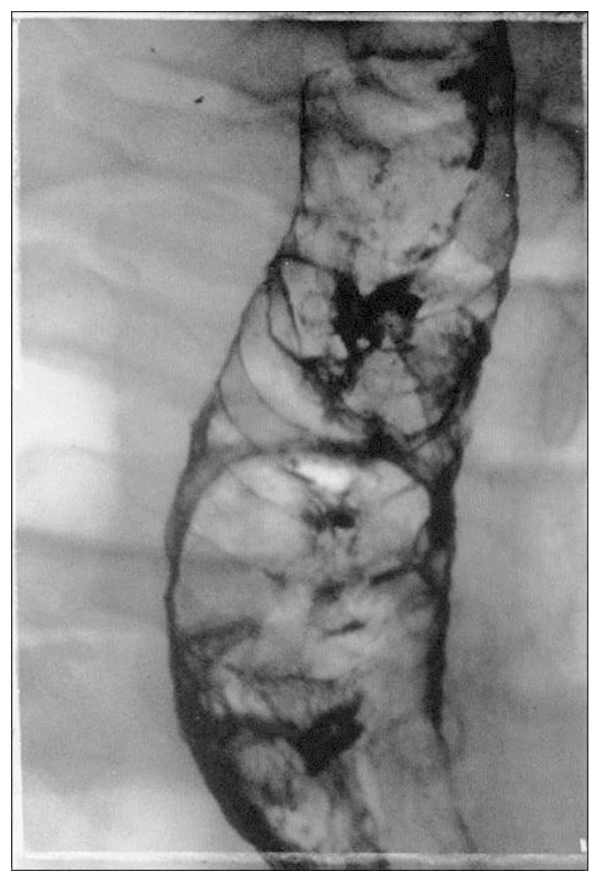

B

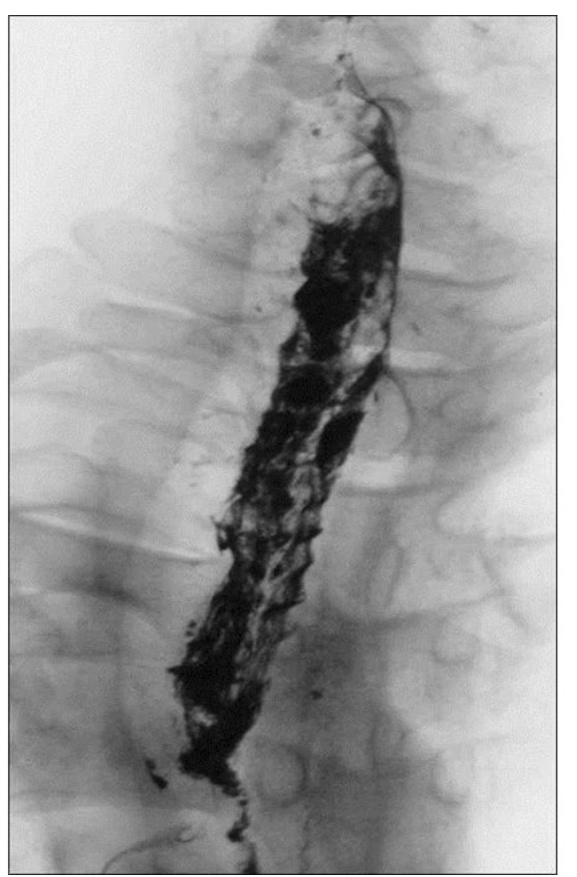

C

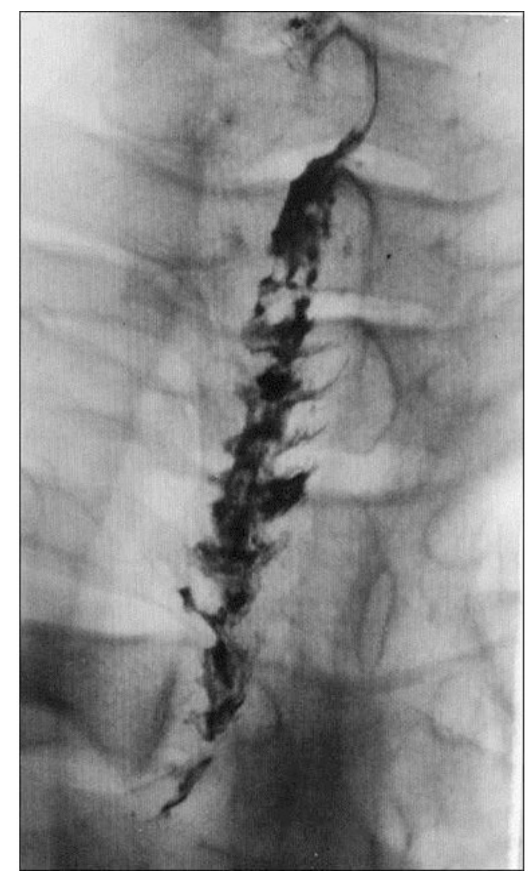

Figure 4. The study group. Trachea: A) tracheal deviation; B) an intensive flow of the tantalum microparticles is seen throughout the trachea length in 20 min after the spray coating; C) the tantalum particles transport is seen on the mucosa of the trachea cartilaginous part.

Thus, depending on the clearance time and morpho functional changes in tuberculosis, COPD and central cancer, 3 degrees of disturbance activity of the ciliated apparatus and MC were established:

$1^{\text {st }}$ degree (24-48 hours) - a moderate disturbance in case of acute inflammatory lung diseases (infiltrative tuberculosis, pneumonia, bronchitis, etc.);

$2^{\text {nd }}$ degree (48-72 hours) - a significant disturbance in case of chronic bronchial and lung pathology (fibrous-cavernous tuberculosis, chronic bronchitis, bronchiectasis, etc.);

$3^{\text {rd }}$ degree ( 72 hours and more) - a severe disturbance (LC).

\section{Conclusion}

1. The dynamic changes in the respiratory tract mucosa after the lungs spraying with tantalum particles is a model of $\mathrm{x}$-ray visualization of the morpho functional activity of the ciliated apparatus and mucociliary clearance both in normal and in patients with tuberculosis, COPD, and LC.

2. The complete clearance time of the mucous membrane from the tantalum particles is normally $15-20$ minutes in the larynx and pharynx, 45-60 minutes - in trachea, and 20-24 hours - in small bronchi. In other words, the speed of particles transport in small bronchi is 60-65 times less than in the larynx and pharynx.

3. It is established that the damaging effect of different particles in the "risk zones" of the mucosa is one of the leading factors that disturbs the regional clearance and contributes to the development of the inflammatory diseases and tumours.

Different time-periods of the mucosa clearance in tuberculosis and LC could be used as one of the reliable criteria in the differential diagnostics (Patent RF No 2173953; in Russian).

\section{References}

1. Iravani J, Melville GN (1982) Mucociliary function in the respiratory tract as influenced by physicochemical factors. In: Encyclopedia of Pharmacology and Therapeutics. Pharmacol Ther B 2: 477-500. [Crossref]

2. Putov NV, Fedoseev GB (1984) Handbook on pulmonology. Leningrad: Meditsina (in Russian).

3. Kobylyanskiy VI (2001) Methods for investigation of mucociliary activity: opportunities and perspectives. Terapevticheskiy arkhiv 3: 73-76.

4. Noreyko SV, Lepshina SM, Grishun Yu A, Shkurat EYu (2002) Mucociliary clearance and bronchial obstruction in patients with epidemic pulmonary tuberculosis. Arkhiv klinicheskoy i eksperimental'noy meditsiny 11: 359-363.

5. Solopov VN (1987) Mucociliary transport in patients with pulmonary obstructive disease. Diss. Moscow.

6. Krishtafovich AA, Koleshko LE, Rebane LE, Ermolova TP (1982) Studying of the tracheal and bronchial mucosa using tantalum powder. Vestnik rentgenologii $i$ radiologii 5: 59-66.

7. Il'ina VA (2014) Morphology of local and general pathology in patients with severe thermo injury. Diss. Saint-Petersburg.

8. Krishtafovich AA, Puchkova TV (2003) Functional evaluation of bronchial mucosa in patients with tuberculosis and other pulmonary diseases. Problemy tuberkuleza I bolezney legkikh 10: 17-20.

9. Krishtafovich AA, Ariel BM (2014) Exocrine gland cysts in tracheal and bronchial walls. Pul'monologiya 3: 99-102.

10. Krishtafovich AA, Ryabinkin VD, Zhukov SY (1996) A device for powder inhalation. Patent RF? 2053801.

11. Krishtafovich AA, Ariel BM, Kozlova NA (2001) A method for radiological differentiation between pulmonary tuberculosis and lung cancer. Patent RF? 2173953.

12. Hasleton HS (1996) Spencer's pathology of the lung. ( $5^{\text {th }}$ edn), McGraw_Hill, New York.

Copyright: (C2019 Krishtafovich AA. This is an open-access article distributed under the terms of the Creative Commons Attribution License, which permits unrestricted use, distribution, and reproduction in any medium, provided the original author and source are credited. 